EPJ Web of Conferences 113, 04013 (2016)

DOI: $10.1051 /$ epjconf/201611304013

C) Owned by the authors, published by EDP Sciences, 2016

\title{
Description of the four-nucleon collisions by including breakup
}

\author{
Rimantas Lazauskas ${ }^{1, a}$ \\ ${ }^{1}$ IPHC, IN2P3-CNRS/Université de Strasbourg BP 28, F-67037 Strasbourg Cedex 2, France
}

\begin{abstract}
Four-nucleon reactions above the breakup threshold are described by solving Faddeev-Yakubovsky equations for the realistic nuclear Hamiltonians. Complex-scaling method is applied in order to simplify the boundary conditions.
\end{abstract}

\section{Introduction}

The accurate description of nuclear interaction remains one of the most important challenges of nuclear physics. The success of this enterprize strongly depends on the ability to provide accurate numerical tools, which could test modern nuclear interaction models in describing nuclear reactions. A proper formalism of three-particle reactions has been introduced by Faddeev in 1961 [1] and few years later generalized by Yakubovsky [2] to any number of particles. Regardless the fact that this formalism enables to handle binary collisions at relative ease, the description of the breakup process into three or more clusters constitutes an important technical and formal challenge. This complexity is mostly due to the complex behavior of the system's wave function in the far asymptotes.

It is quite obvious that a direct approach based on the explicit treatment of boundary conditions becomes overcomplex already for the systems containing more than three particles. Recently a formalism [3], which combines Faddeev-Yakubovsky (FY) equations [2] and complex scaling method [4], has been developed in order to solve three- and four-body scattering problems above the three-particle breakup threshold. This formalism relies on trivial boundary conditions, is easy to handle but still provides accurate results [3].

In this manuscript the latest developments in the four-nucleon sector are reviewed. This includes calculations for $\mathrm{n}^{3}{ }^{3} \mathrm{H}$ and $\mathrm{p}^{3} \mathrm{He}$ scattering above the three- and four-nucleon breakup thresholds.

\section{The formalism}

A detailed overview of complex scaling method has been recently presented in [5]. This method, introduced by Nuttall and Cohen [4], might be employed to solve Scrödinger equation directly. Nevertheless, in this study configuration space FY equations [2] are used. FY equations are built to separate the asymptotes of the binary channels and therefore are advantageous in describing systems, where binary collisions are dominant. After separation of the incoming plane wave, FY equations formulated for a system of four identical fermions reads:

$$
\begin{gathered}
K(\vec{x}, \vec{y}, \vec{z})=K^{\text {out }}(\vec{x}, \vec{y}, \vec{z})+K^{\text {in }}(\vec{x}, \vec{y}, \vec{z}) \\
H(\vec{x}, \vec{y}, \vec{z})=H^{\text {out }}(\vec{x}, \vec{y}, \vec{z})+H^{\text {in }}(\vec{x}, \vec{y}, \vec{z}) .
\end{gathered}
$$

\footnotetext{
a e-mail: rimantas.lazauskas@iphc.cnrs.fr
} 


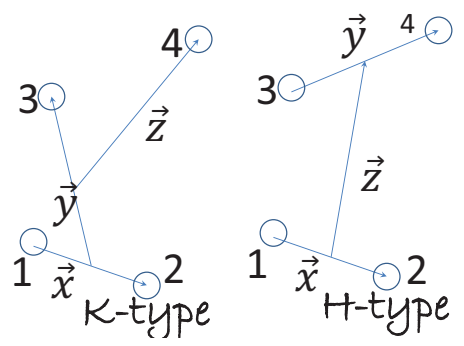

Figure 1. The FY components $K_{12,3}^{4}$ and $H_{12}^{34}$ for a given particle ordering. As $z \rightarrow \infty$, the $K$ components absorb asymptotes of $3+1$ particle channels, whereas the $H$ components absorb asymptotes of $2+2$ channels.

Four-body FY equations involve components of two types, $\mathrm{K}$ and $\mathrm{H}$. In their $z \rightarrow \infty$ asymptotes these components separate respectively outgoing $\left(K^{\text {out }}, H^{\text {out }}\right)$ and incoming $\left(K^{\text {in }}, H^{\text {in }}\right)$ waves of $(3+1,2+2)$ particle channels, see fig. 1. These components are the solutions of the inhomogeneous FY equations:

$$
\begin{aligned}
\left(E-H_{0}-V_{12}\right) K^{\text {out }}- & V_{12}\left(P^{+}+P^{-}\right)\left[(1+Q) K^{\text {out }}+H^{\text {out }}\right] \\
& =V_{12}\left(P^{+}+P^{-}\right)\left[H^{\text {in }}+Q K^{\text {in }}\right], \\
\left(E-H_{0}-V_{12}\right) H^{\text {out }}- & V_{12} \tilde{P}\left[(1+Q) K^{\text {out }}+H^{\text {out }}\right]=V_{12} \tilde{P}\left[(1+Q) K^{\text {in }}\right],
\end{aligned}
$$

here $P^{-}=P_{23} P_{12}, P^{+}=P_{12} P_{23}, Q=P_{34}$ and $\tilde{P}=P_{13} P_{24}$ are particle permutation operators.

Then the complex scaling method is applied. I.e. one acts on the last set of equations with a complex-scaling operator:

$$
\widehat{S}=e^{i \theta r \frac{\partial}{\partial r}}=e^{i \theta\left(x \frac{\partial}{\partial x}+y \frac{\partial}{\partial y}+z \frac{\partial}{\partial z}\right)},
$$

where parameter $\theta$ is often referred as complex scaling angle. The complex scaled FY equations are solved to determine the transformed FY components $\tilde{K}^{\text {out }}=\widehat{S} K^{\text {out }}$ and $\tilde{H}^{\text {out }}=\widehat{S} H^{\text {out }}$. In particular, the complex scaling operator transforms outgoing waves into square integrable functions $\tilde{K}^{\text {out }}$ (or $\tilde{H}^{\text {out }}$ ) if a complex scaling angle is chosen in the interval $0<\theta<\pi$. At the same time functions $\tilde{K}^{\text {in }}$ (or $\tilde{H}^{i n}$ ) become exponentially divergent. Nevertheless these functions appear only in inhomogeneous term of the FY equations (3-4) premultiplied with potential energy operator, and thus if interactions are exponentially bound the kernel of the complex scaled equations becomes compact ${ }^{1}$.

The transformed FY equations are solved using standard techniques, developed in our preceding studies, see [3] and references therein. Namely, the spin-isospin as well as angular dependence of the FY components is expanded using partial-waves. The radial dependence of the complex-scaled FY components $\tilde{K}^{\text {out }}$ and $\tilde{H}^{\text {out }}$ is expanded on the Lagrange-Laguerre basis and a system of integrodifferential equations is transformed into linear algebra problem by using Lagrange-mesh method [6]. For neutron energies below $30 \mathrm{MeV}$ numerical convergence is achieved by including partial-waves with angular momenta $\max \left(l_{x}, l_{y}, l_{z}\right) \leq 4$, also 3D Lagrange-mesh of $\sim 30^{3}$ points is required to describe radial dependence of the FY components $\left(\tilde{K}^{\text {out }}, \tilde{H}^{\text {out }}\right)$. This leads to linear systems of $\sim 10^{8}$ equations, which are solved using iterative linear algebra methods. Scattering amplitudes, including breakup ones, are calculated from transformed solutions $\tilde{K}^{\text {out }}\left(\right.$ or $\left.\tilde{H}^{\text {out }}\right)$ by employing integral relations $[3,5]$.

\footnotetext{
${ }^{1}$ One should note that when considering scattering in $A>2$ particle system additional condition is implied on complex scaling angle in order to guarantee compactness of the kernel [5].
} 

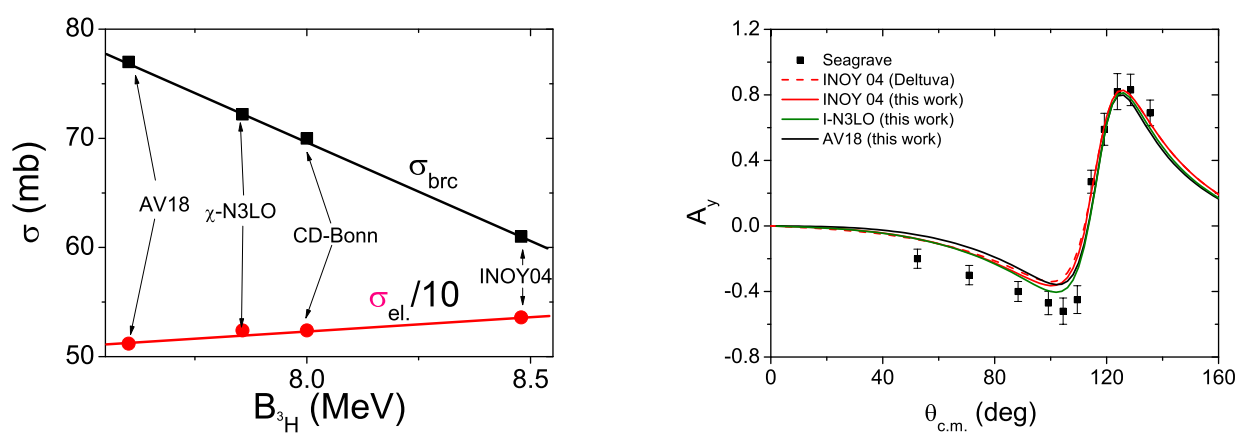

Figure 2. Correlation of $\mathrm{n}^{-3} \mathrm{H}$ total elastic and breakup cross sections with the model predicted ${ }^{3} \mathrm{H}$ binding energy (left panel). Neutron ${ }^{3} \mathrm{H}$ analyzing powers $A_{y}$ (right panel). Calculations have been performed for incident neutrons with laboratory energy of $22.1 \mathrm{MeV}$. Experimental results on analyzing powers are from J. Seagrave $e t$ al. [9].

\section{Selected results}

The complex scaling method enables to consider $4 \mathrm{~N}$ system above the breakup threshold employing fully realistic NN interaction models. First this method was applied to explore neutron scattering on ${ }^{3} \mathrm{H}$ nucleus, a system which does not involve Coulomb interaction. In particular, the region of neutron laboratory energies from 14 to $22 \mathrm{MeV}$ has been explored, using INOY04, $\chi \mathrm{N} 3 \mathrm{LO}$ and AV18 NN interaction models $[3,5]$. Few years earlier the same system had been examined by A. Deltuva et al. [7], within the momentum space formulation of the complex-energy method. An excellent agreement is found between two very different approaches, reaching three-digit accuracy for the calculated observables. By analyzing different model predictions, a correlation pattern has been established between both, the total elastic and the total break up cross section, and the predicted ${ }^{3} \mathrm{H}$ binding energy (see fig. 2). Moreover, once trinucleon binding energy is reproduced, theoretical prediction for the total cross section complies with the experimental data. Neutron analyzing powers also agree reasonably well with the experimental data, nevertheless the theoretical predictions systematically underestimate experimental data at the minima.

In the previous studies on 3-body system a possibility to implement the repulsive Coulomb interaction has been demonstrated, when describing nuclear reactions above the breakup threshold $[3,5]$. The formalism essentially remains the same, only free in(out)going waves should be replaced with the Coulomb ones. As discussed in [5], small approximation is however involved in this case, which consist in neglecting long-range Coulomb polarization terms between the separating fragments.

The inclusion of the repulsive Coulomb interaction in the complex-scaling formalism allows to consider proton scattering on ${ }^{3} \mathrm{He}$. This reaction has been explored for the protons with energy from 8.5 MeV and 19.4 MeV, using realistic Hamiltonian based on AV18 and INOY04 NN interaction models. The obtained results are compared with the calculations of A. Deltuva et al. [8] and the experimental data from ref. [10]. This time the agreement between the two calculations is slightly worse than for a Coulomb-free case, small discrepancy of order 5\% between two theoretical predictions is observed at the backward angles. It is difficult to trace the origin of this discrepancy due to the fact that the final cross section is a result of the strong interference of separate partial waves. Nevertheless, a good overall agreement is observed with the existent experimental cross sections for the INOY04 Hamiltonian. On contrary the AV18 interaction model slightly underestimates the differential cross 

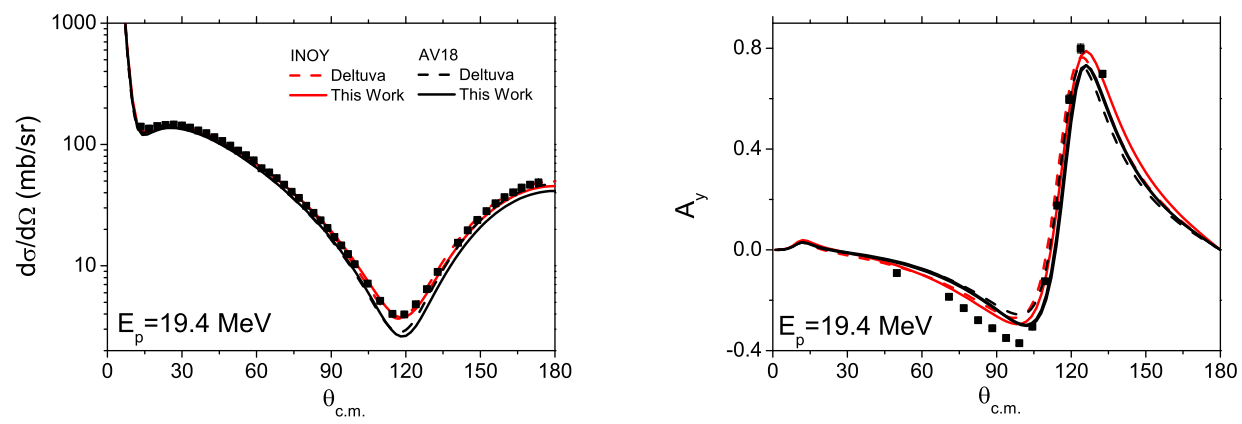

Figure 3. Calculated $\mathrm{p}-{ }^{3} \mathrm{He}$ elastic differential cross sections and analyzing powers for the protons of laboratory energy 19.4 MeV. Calculated values are compared with ones from ref. [8] and with the experimental data from ref. [10].

section at the minima, this is visibly related with ${ }^{3} \mathrm{He}$ nucleus being underbound by this model. The proton- ${ }^{3} \mathrm{He}$ analyzing powers are similar to the neutron- ${ }^{3} \mathrm{H}$ ones, in particular the theoretical predictions underestimate experimental data at the minima.

\section{Acknowledgements}

This work was granted access to the HPC resources of TGCC and IDRIS under the allocation 2015-x2015056006 made by GENCI. We thank the staff members of the TGCC and IDRIS for their constant help.

\section{References}

[1] L. D. Faddeev, Zh. Eksp. Teor. Fiz. 39, 1459 (1960). [Sov. Phys. JETP 12, 1014 (1961)].

[2] O. A. Yakubovsky, Sov. J. Nucl. Phys. 5, 937 (1967).

[3] R. Lazauskas and J. Carbonell, Phys. Rev. C 84, 034002 (2011); R. Lazauskas, Phys. Rev. C 91, 041001(R) (2015).

[4] J. Nuttall and H. L. Cohen, Phys. Rev. 188, 1542 (1969).

[5] A. Deltuva et al., PPNP 74, 55 (2014).

[6] D. Baye, Phys. Rep. 565 (2015) 1.

[7] A. Deltuva and A. C. Fonseca, Phys. Rev. C 86, 011001 (2012).

[8] A. Deltuva and A. C. Fonseca, Phys. Rev. C 87, 054002 (2013).

[9] J. Seagrave et al., Annals of Physics 74, 250 (1972).

[10] S. D. Baker, et al., Nucl. Phys. A160, 428 (1971). 